\title{
A GENERALIZED FOURIER TRANSFORMATION FOR $L_{1}(G)$-MODULES
}

\author{
TENG-SUN LIU, ARNOUD C. M. VAN ROOIJ and JU-KWEI WANG
}

(Received 9 November 1982)

Communicated by G. H. Price

\begin{abstract}
Let $G$ be a compact abelian group with dual $\hat{G}$ and let $K$ be a Banach $L_{1}(G)$-module. We introduce the notion of character convolution transformation of $K$ which reduces to ordinary Fourier or FourierStieltjes transformation when $K$ is one of the spaces $L_{p}(G), M(G)$. We show that the question of what maps $\hat{G} \rightarrow K$ extend to multipliers of $K$ is a question of asking for descriptions of the character convolution transforms. In this setting some results of Helson-Edward and Schoenberg-Eberlein find generalizations, as do some classical results, including the inversion formula and the Parseval relation. We then apply these results to transformation groups, obtaining a variant of a theorem of Bochner and an extension of a theorem of Ryan.
\end{abstract}

1980 Mathematics subject classification (Amer. Math. Soc.): 42 A 20, 43 A 25.

\section{Introduction}

Let $G$ be a compact abelian group.

As is well-known, $L_{1}(G)$ is a commutative Banach algebra under convolution. A Banach $L_{1}(G)$-module $K$ (see $\left.[4 ; 32.14]\right)$ is a Banach space $K$ that is also a module over the ring $L_{1}(G)$, such that (if $*$ denotes the module multiplication)

$$
f * \alpha x=\alpha f * x=\alpha(f * x) \quad\left(\alpha \in \mathbf{C} ; f \in L_{1}(G) ; x \in K\right)
$$

and

$$
\|f * x\| \leqslant\|f\|\|x\| \quad\left(f \in L_{1}(G) ; x \in K\right) .
$$

Under convolution, $L_{p}(G)(1 \leqslant p \leqslant \infty), C(G)$ and $M(G)$ are Banach $L_{1}(G)$-modules. More examples are given in [4; Section 32], [1; Section 4] and in Section 3 of this paper.

(c) 1984 Australian Mathematical Society 0263-6115/84\$A2.00+0.00 
We are going to occupy ourselves with the following two problems, that turn out to be closely related. Let $K$ be a Banach module over $L_{1}(G)$.

$(\alpha)$ Introduce an analog of the Fourier-Stieltjes transformation that reduces to the ordinary Fourier or Fourier-Stieltjes transformation if $K$ is one of the spaces $L_{p}(G), M(G)$.

( $\beta$ ) A multiplier of $K$ is a continuous module homomorphism $L_{1}(G) \rightarrow K$. (See [5].) Consider the dual group $\hat{G}$ of $G$ as a subset of $L_{1}(G)$. As $\hat{G}$ spans a dense linear subspace of $L_{1}(G)$, a map $\hat{G} \rightarrow K$ has at most one continuous linear extension $L_{1}(G) \rightarrow K$. What maps $\hat{G} \rightarrow K$ extend to multipliers of $K$ ?

For $K=L_{1}(G)$ the relation between $(\alpha)$ and $(\beta)$ is easy to describe: by Wendel's characterization of the multipliers of $L_{1}(G)[4 ; 35.5]$ one sees that a map $\phi: \hat{G} \rightarrow L_{1}(G)$ extends to a multiplier if and only if there exists a $\mu \in M(G)$ such that $\phi(\gamma)=\hat{\mu}(\gamma) \gamma$ for all $\gamma \in \hat{G}$.

Our notations are mostly those used by E. Hewitt and K. A. Ross in [4].

Throughout the paper, $G$ is a compact abelian group whose dual group is denoted $\hat{G}$, and $K$ is a Banach $L_{1}(G)$-module. Both convolution $L_{1}(G) \times L_{1}(G)$ $\rightarrow L_{1}(G)$ and module multiplication $L_{1}(G) \times K \rightarrow K$ are indicated by *. The Haar integral of $f \in L_{1}(G)$ are written $\int f(s) d s$.

$K$ is called order-free if for every $x \in K, x \neq 0$ there exists an $f \in L_{1}(G)$, $f * x \neq 0$. The trigonometric polynomials (that is, the linear combinations of characters) form a dense linear subspace of $L_{1}(G)$. It follows that $K$ is order-free if and only if for every $x \in K, x \neq 0$ there exists a $\gamma \in \hat{G}$ such that $\gamma * x \neq 0$.

By $[4 ; 32.22]$ the products $f * x\left(f \in L_{1}(G) ; x \in K\right)$ form a closed linear subspace $K_{\text {abs }}$ of $K$. This $K_{\text {abs }}$ is a Banach $L_{1}(G)$-submodule of $K$. As $L_{1}(G)$ has an approximate identity $K_{\text {abs }}$ is order-free. In particular, if $x, y \in K_{\text {abs }}$ and if $\gamma * x=\gamma * y$ for all $\gamma \in \hat{G}$, then $x=y$.

$K$ is said to be absolutely continuous if $K=K_{\text {abs }}$. Examples: $L_{p}(G)(1 \leqslant p<\infty)$ and $C(G)$ are absolutely continuous $[4 ; 32.20$ and 32.31$] ; L_{\infty}(G)$ is not $[4 ; 20.16]$; neither is $M(G)[4 ; 19.18]$.

For $f \in L_{1}(G)$ define $f^{*} \in L_{1}(G)$ by $f^{*}(s)=f\left(s^{-1}\right)(s \in G)$. We make the conjugate space $K^{*}$ of $K$ into a Banach $L_{1}(G)$-module by defining

$$
(x, f * h)=\left(f^{*} * x, h\right) \quad\left(f \in L_{1}(G) ; x \in K ; h \in K^{*}\right) .
$$

(We might just as well have taken $f * x$ instead of $f^{*} * x$. However, $f^{*} * x$ is more appropriate in the more general situation where one does not confine ones attention to compact abelian group $G$.) 
If by a similar formula one puts a module structure on $K^{* *}$, the natural map $K \rightarrow K^{* *}$ is a module homomorphism.

For $h \in K^{*}$ we have $L_{1}(G) * h=\{0\}$ if and only if $h$ vanishes on $K_{\mathrm{abs}}$. Hence, $K^{*}$ is order-free if and only if $K$ is absolutely continuous.

The continuous linear module homomorphisms $L_{1}(G) \rightarrow K$ are called the multipliers of $K$; they form a Banach space Mult $K$. Every $x \in K$ induces a $T_{x} \in$ Mult $K$ by

$$
T_{x} f=f * x \quad\left(f \in L_{\mathrm{l}}(G)\right) .
$$

This $T: K \rightarrow$ Multi $K$ is injective if and only if $K$ is order-free. In particular, the restriction of $T$ to $K_{\text {abs }}$ is injective (it is also an isometry; see [3; 5.1(iv)]).

1.1 Lemma. For $\gamma \in \hat{G}$, define $K_{\gamma}=\gamma * K(=\{\gamma * x \mid x \in K\})$. Then

$$
\begin{aligned}
K_{\gamma} & =\{x \in K \mid \gamma * x=x\} \\
& =\left\{x \in K \mid f * x=\hat{f}(\gamma) x \text { for every } f \in L_{1}(G)\right\} .
\end{aligned}
$$

$K_{\gamma}$ is closed linear subspace of $K$. The map $x \mapsto \gamma * x$ is a continuous idempotent map of $K$ onto $K_{\gamma}$. If $\beta \in \hat{G}, \beta \neq \gamma$, then $\beta * K_{\gamma}=\{0\}$. Further,

$$
K_{\text {abs }}=\text { clo } \sum_{\gamma \in \hat{G}} K_{\gamma} \text {. }
$$

Proof. We only prove the last sentence; the proof of the rest is simple. Obviously $\gamma * K \subset K_{\text {abs }}$ for all $\gamma \in \hat{G}$, so $K_{\text {abs }} \supset$ clo $\Sigma\left\{K_{\gamma} \mid \gamma \in \hat{G}\right\}$. Conversely, $\hat{G}$ spans a dense linear subspace of $L_{1}(G)$; hence if $x \in K$, then $\{\gamma * x \mid \gamma \in \hat{G}\}$ spans a dense linear subspace of $L_{1}(G) * x$. It follows that clo $\Sigma_{\gamma} K_{\gamma} \supset K_{\text {abs }}$.

For Hilbert spaces we have a more detailed knowledge:

1.2 TheOREM. Let $K$ be a Hilbert space; let $\langle$,$\rangle be its inner product. Then$

$$
\langle f * x, y\rangle=\left\langle x, \bar{f}^{*} * y\right\rangle \quad\left(x, y \in K ; f \in L_{1}(G)\right) .
$$

If $\beta, \gamma$ are distinct, then $K_{\beta} \perp K_{\gamma}$. For each $\gamma$, the map $x \mapsto \gamma * x$ is the orthogonal projection of $K$ onto $K_{\gamma}$. For every $x \in K$ the sum $\Sigma_{\gamma \in \hat{G}} \gamma * x$ converges in the sense of the norm. The map $x \mapsto \Sigma \gamma * x$ is the orthogonal projection of $K$ onto $K_{\mathrm{abs}}$. Its kernel is

$$
\left\{x \in K \mid L_{1}(G) * x=\{0\}\right\} .
$$

Proof. Take $\gamma \in \hat{G}$, put $P x=x-\gamma * x$ for $x \in K$. Then $P=P^{2}$ and $\|I-P\|$ $\leqslant 1$. Let $P(K)^{\perp}=\{x \mid x \perp P(K)\}$. If $x \in P(K)^{\perp}$, then $x \perp P x$, so

$$
\|x\|^{2}+\|P x\|^{2}=\|x-P x\|^{2} \leqslant\|x\|^{2} .
$$


Hence, $P(K)^{\perp} \subset P^{-1}(0)$. Conversely, every $x \in P^{-1}(0)$ can be written as $x=y+z$ where $y \in P(K), z \in P(K)^{\perp}$. (Notice that $P(K)=(I-P)^{-1}(0)$ is closed.) Then $z \in P^{-1}(0)$, so $y=x-z \in P^{-1}(0)$. But $y \in P(K)$ and $P=P^{2}$ : it follows that $y=0$ and $x=z \in P(K)^{\perp}$. Therefore, $P(K)^{\perp}=P^{-1}(0)$. Consequently, $P$ is an orthogonal projection. Then so is the map $x \mapsto \gamma * x$. We see that

$$
\langle f * x, y\rangle=\left\langle x, \bar{f}^{*} * y\right\rangle \quad(x, y \in K)
$$

if $f \in \hat{G}$. The same formula holds, by linearity, for all trigonometric functions $f$, and, by continuity, for all $f \in L_{1}(G)$. The rest is easy.

Note. The formula

$$
T_{f} x=f * x \quad\left(f \in L_{1}(G) ; x \in K\right)
$$

yields a correspondence between the module structures $*$ on $K$ and the representations $T$ of $L_{1}(G)$ in $K$ for which $\left\|T_{f}\right\| \leqslant\|f\|\left(f \in L_{1}(G)\right)$. By the above theorem, every such representation is a * -representation.

1.3 LemMa. Let $K$ be a Banach $L_{1}(G)$-module. For a map $T: L_{1}(G) \rightarrow K$ the following conditions are equivalent.

(i) $T \in$ Mult $K$.

(ii) $T$ is linear and continuous; $T \gamma \in K_{\gamma}$ for every $\gamma \in \hat{G}$.

(iii) $T(f * g)=f * T g$ for all $f, g \in L_{1}(G)$.

Proof. (i) $\Rightarrow$ (iii) is obvious.

(ii) $\Rightarrow$ (i). For $\gamma \in \hat{G}$ we have $\gamma * T \gamma=T \gamma=T(\gamma * \gamma)$. If $\beta, \gamma \in \hat{G}$ are distinct, then $\beta * T \gamma=0=T(\beta * \gamma)$. Hence, $f * T g=T(f * g)$ if $f, g \in L_{1}(G)$ are trigonometric polynomials. These forming a dense subspace of $L_{1}(G)$ we find $f * T g=$ $T(f * g)$ for all $f, g \in L_{1}(G)$.

(iii) $\Rightarrow$ (ii). (See [9].) Clearly $T$ maps $L_{1}(G)_{\text {abs }}$ into $K_{\text {abs. }}$. But $L_{1}(G)_{\text {abs }}=L_{1}(G)$ [4;32.30], so the range of $T$ lies in $K_{\text {abs. }}$. For all $f, g \in L_{1}(G), f * T g=T(f * g)=$ $T(g * f)=g * T f$. If $g_{1}, g_{2} \in L_{1}(G)$ and $c_{1}, c_{2} \in \mathbf{C}$, then for all $f \in L_{1}(G)$

$$
\begin{aligned}
f *\left[c_{1} T g_{1}+c_{2} T g_{2}\right] & =c_{1} f * T g_{1}+c_{2} f * T g_{2} \\
& =c_{1} g_{1} * T f+c_{2} g_{2} * T f=\left(c_{1} g_{1}+c_{2} g_{2}\right) * T f \\
& =f * T\left(c_{1} g_{1}+c_{2} g_{2}\right) .
\end{aligned}
$$

As $c_{1} T g_{1}+c_{2} T g_{2}-T\left(c_{1} g_{1}+c_{2} g_{2}\right) \in K_{\text {abs }}$ and $K_{\text {abs }}$ is order-free, it follows that $c_{1} T g_{1}+c_{2} T g_{2}=T\left(c_{1} g_{1}+c_{2} g_{2}\right)$. Thus, $T$ is linear. The continuity of $T$ is proved with the aid of the Closed Graph Theorem. Let $f_{1}, f_{2}, \ldots$ be a sequence in $L_{1}(G)$ such that $\lim f_{n}=0$ while $\lim T f_{n}$ exists in $K$. Then $\lim T f_{n} \in K_{\text {abs }}$, and for all $g \in L_{1}(G), g * \lim T f_{n}=\lim g * T f_{n}=\lim f_{n} * T g=0$. Hence, $\lim T f_{n}=0$ and $T$ is continuous. Finally, for $\gamma \in \hat{G}$ one has $T \gamma=T(\gamma * \gamma)=\gamma * T \gamma \in K_{\gamma}$. 
The implication (ii) $\Rightarrow$ (i) gives the situation a new perspective. Apparently, a map $\phi: \hat{G} \rightarrow K$ extends to a multiplier if and only if $\phi \in \Pi_{\gamma} K_{\gamma}$ and $\phi$ admits a continuous linear extension $L_{1}(G) \rightarrow K$. The question remains: what $\phi \in \Pi_{\gamma} K_{\gamma}$ do admit such an extension?

For $T \in$ Mult $K$ we denote by $\tilde{T}$ the restriction of $T$ to $\hat{G}$. $T$ is determined by $\tilde{T}$, since the characters of $G$ span a dense linear subspace of $L_{1}(G)$. thus,

Every $x \in K$ determines a multiplier $T_{x}: f \mapsto f * x$. Instead of $\tilde{T}_{x}$ we write $\tilde{x}$;

$$
\tilde{x}_{\gamma}=\tilde{x}(\gamma)=\gamma * x \quad(\gamma \in \hat{G} ; x \in K) .
$$

If $K=L_{1}(G)$, then $\tilde{x}_{\gamma}=\hat{x}(\gamma) \gamma$, so $\tilde{x}$ actually is the Fourier "series" of $x$. For arbitrary modules $K$ we call $\tilde{x}$ the character convolution transform of $x$.

We know by Wendel's theorem $[4 ; 35.5]$ that Mult $L_{1}(G)$ may be identified with $M(G)$. If $T \in$ Mult $L_{1}(G)$ corresponds to $\mu \in M(G)$, then $\tilde{T}(\gamma)=\hat{\mu}(\gamma) \gamma$. Thus, the map $T \mapsto \tilde{T}$ can be viewed as a generalization of the Fourier-Stieltjes transformation.

We see now how our problems $(\alpha)$ and $(\beta)$ converge: the character convolution transformation is an answer to $(\alpha)$, and $(\beta)$ asks for descriptions of character convolution transforms.

A few simple observations:

2.1 LemMa. For $x \in K$,

$$
\tilde{x}=0 \text { if and only if } L_{1}(G) * x=\{0\} .
$$

In particular, if $x, y \in K_{\mathrm{abs}}$ and $\tilde{x}=\tilde{y}$, then $x=y$.

2.2 LEMMA. We have the relations

$$
(T f)^{\tilde{}}=\hat{f} \tilde{T} \quad\left(f \in L_{1}(G) ; T \in \text { Mult } K\right)
$$

and

$$
(f * x)^{\tilde{}}=\hat{f} \tilde{x} \quad\left(f \in L_{1}(G) ; x \in K\right) .
$$

The following extension of the Helson-Edwards Theorem [7; 3.8.1] holds.

2.3 TheOREM. $\phi \in \Pi_{\gamma} K_{\gamma}$ can be extended to a multiplier of $K$ if and only if $\hat{f} \phi \in \tilde{K}$ for every $f \in L_{1}(G)$. (We put $\tilde{K}=\{\tilde{x} \mid x \in K\}$.) 
Proof. If $T \in$ Mult $K$ and $\phi=\tilde{T}$, then for every $f \in L_{1}(G)$ we have $\hat{f} \phi=\hat{f} \tilde{T}$ $=(T f) \tilde{H} \in \tilde{K}$. Conversely, suppose $\phi \in \Pi_{\gamma} K_{\gamma}$ and $\hat{f} \phi \in \tilde{K}$ for all $f \in L_{1}(G)$. Every $f \in L_{1}(G)$ can be written as $f=f_{1} * f_{2}$ with certain $f_{1}, f_{2} \in L_{1}(G)$; then $\hat{f} \phi=\hat{f}_{1}\left(\hat{f}_{2} \phi\right) \in \hat{f}_{1} \tilde{K}=\left(f_{1} * K\right)^{\sim} \subset\left(K_{\text {abs }}\right)^{-}$. By Lemma 2.1 , for every $f \in L_{1}(G)$ there is a unique $T f \in K_{\text {abs }}$ such that $\hat{f} \phi=(T f)^{\tilde{y}}$. If $f, g \in L_{1}(G)$, then $(f * T g)$ $=\hat{f}(T g)^{\tilde{f}}=\hat{f} \hat{g} \phi=(f * g)^{\hat{\phi}} \phi=(T(f * g))^{\tilde{y}}$, so $f * T g=T(f * g)$. By Lemma 1.3 $T$ is a multiplier of $K$. Further, $(T \gamma)^{\sim}=\hat{\gamma} \phi=\left(\phi_{\gamma}\right)^{\tilde{y}}$, so $T \gamma=\phi_{\gamma}$ for every $\gamma \in \hat{G}$.

Another characterization displays a certain analogy with the Schoenberg-Ebelein Theorem [4; 33.20], [7; 1.9.1].

2.4 THEOREM. $\phi \in \Pi_{\gamma} K_{\gamma}$ can be extended to a multiplier of $K$ if and only if there exists a constant $c$ such that

$$
\left\|\sum_{i=1}^{n} c_{i} \phi_{\gamma_{i}}\right\| \leqslant c\left\|\sum_{i=1}^{n} c_{i} \gamma_{i}\right\|_{1}
$$

for every trigonometric polynomial $\Sigma c_{i} \gamma_{i}$ on $G$.

Proof. If $T \in$ Mult $K$ and $\phi=\tilde{T}$, then for every trigonometric polynomial $\Sigma c_{i} \gamma_{i}$ we have

$$
\left\|\sum c_{i} \phi_{\gamma_{i}}\right\|=\left\|\sum c_{i} T\left(\gamma_{i}\right)\right\|=\left\|T\left(\sum c_{i} \gamma_{i}\right)\right\| \leqslant\|T\|\left\|\sum c_{i} \gamma_{i}\right\|_{1} .
$$

Conversely, if $\phi \in \Pi_{\gamma} K_{\gamma}$ and if there exists a constant $c$ such that (*) holds for every trigonometric polynomial, then (as the trigonometric polynomials are dense in $L_{1}(G)$ ) we have a continuous linear $T: L_{1}(G) \rightarrow K$ such that $T\left(\Sigma c_{i} \gamma_{i}\right)=\Sigma c_{i} \phi_{\gamma_{i}}$ for all $\Sigma c_{i} \gamma_{i}$. In particular, $T \gamma=\phi_{\gamma}$ for $\gamma \in \hat{G}$. Then $T \in$ Mult $K$ by the implication (ii) $\rightarrow$ (i) of Lemma 1.3.

Note. A better analogy with the Schoenberg-Eberlein Theorem would be obtained if in (*) we could replace the $L_{1}$-norm by the $L_{\infty}$-norm. This change, however, would make the theorem false, as one sees from the example $K=C(G)$, $\phi_{\gamma}=\gamma$.

The following theorem, and also 2.9, are inversion theorems, stating that certain elements of a module are the sums of their character convolution transforms, as many functions of $L_{\mathrm{J}}(G)$ are the sums of their Fourier series. $F$ denotes the directed set of all finite subsets of $\hat{G}$.

2.5 THeOREM. Let $\phi \in \Pi_{\gamma} K_{\gamma}$ be so that the net $\left(\Sigma_{\gamma \in \Delta} \phi_{\gamma}\right)_{\Delta \in F}$ is bounded. Then $\phi$ can be extended to a multiplier $T$ of $K$. For all $f \in L_{1}(G)$ we have

$$
T f=\sum_{\gamma \in \hat{G}} f * \phi_{\gamma}
$$


Proof. for $\Delta \in F$ put $\phi_{\Delta}=\Sigma_{\gamma \in \Delta} \phi_{\gamma}$. Let $c=\sup _{\Delta \in F}\left\|\phi_{\Delta}\right\|$. If $\Sigma c_{i} \gamma_{i}$ is a trigonometric polynomial, then for $\Delta=\left\{\gamma_{1}, \ldots, \gamma_{n}\right\}$ we have

$$
\left\|\sum c_{i} \phi_{\gamma_{i}}\right\|=\left\|\sum c_{i} \gamma_{i} * \phi_{\Delta}\right\| \leqslant c\left\|\sum c_{i} \gamma_{i}\right\|_{1}
$$

so $\phi$ is extendable to a multiplier $T$. Furthermore, if $\Sigma c_{i} \gamma_{i}$ is a trigonometric polynomial, then for $\Delta \supset\left\{\gamma_{1}, \ldots, \gamma_{n}\right\}$ we have

$$
T\left(\sum c_{i} \gamma_{i}\right)=\sum c_{i} \phi_{\gamma_{i}}=\sum c_{i} \gamma_{i} * \phi_{\Delta} \text {. }
$$

If $g \in L_{1}(G)$ and $\varepsilon>0$, there is a trigonometric polynomial $f \in L_{1}(G)$ such that $\|f-g\|_{1}<\varepsilon$; there is a $\Delta_{0} \in F$ such that $T f=f * \phi_{\Delta}$ for $\Delta \supset \Delta_{0}$. Then, for $\Delta \supset \Delta_{0}$,

$$
\left\|T g-g * \phi_{\Delta}\right\| \leqslant\|T(g-f)\|+\left\|(f-g) * \phi_{\Delta}\right\| \leqslant \varepsilon(\|T\|+c)
$$

Hence, $T g=\lim _{\Delta \in F} g * \phi_{\Delta}=\lim _{\Delta \in \mathrm{F}} \Sigma_{\gamma \in \Delta} g * \phi_{\gamma}$.

The following is another variant of the Schoenberg-Eberlein criterion.

2.6 THEOREM. The following conditions on $\phi \in \Pi_{\gamma}\left(K^{*}\right)_{\gamma}$ are equivalent:

(i) $\phi \in\left(K^{*}\right)^{\sim}$.

(ii) $\phi$ can be extended to a multiplier of $K^{*}$.

(iii) There exists a constant $c$ such that for every positive integer $n$ and for all $\gamma_{1}, \ldots, \gamma_{n} \in \hat{G}$ and $x_{1}, \ldots, x_{n} \in K$,

$$
\left|\sum_{i=1}^{n}\left(x_{i}, \phi_{\gamma_{i}}\right)\right| \leqslant c\left\|\sum \gamma_{i} * x_{i}\right\| .
$$

Proof. (i) $\Rightarrow$ (ii). If $h \in K^{*}$ and $\phi=\tilde{h}$, then $f \mapsto f * h$ is a multiplier of $K^{*}$ that is an extension of $\phi$.

(ii) $\Rightarrow$ (iii). Let $\phi=\tilde{T}, T \in$ Mult $K^{*}$. We identify $L_{l}(G)^{*}$ with $L_{\infty}(G)$. It is not difficult to verify that the module operation on $L_{1}(G)^{*}$ corresponds to the module operation on $L_{\infty}(G)$. In particular, for $f \in L_{1}(G)$ and $h \in L_{\infty}(G)$,

$$
(f, h)=\left(f^{*} * h\right)(e) \text {, }
$$

$e$ denoting the unit element of $G$. Now $T$ induces a continuous linear $S$ : $K \rightarrow L_{\infty}(G)$ by

$$
(f, S x)=(x, T f) \quad\left(f \in L_{1}(G) ; x \in K\right) .
$$

For $f, g \in L_{1}(G)$ and $x \in K$.

$$
\begin{aligned}
(f, g * S x) & =\left(g^{*} * f, S x\right)=\left(x, T\left(g^{*} * f\right)\right) \\
& =\left(x, g^{*} * T f\right)=(g * x, T f)=(f, S(g * x))
\end{aligned}
$$

so that $g * S x=S(g * x)$. Now take $\gamma_{1}, \ldots, \gamma_{n} \in \hat{G}$ and $x_{1}, \ldots, x_{n} \in K$. 


$$
\begin{aligned}
\left|\sum\left(x_{i}, \phi_{\bar{\gamma}_{i}}\right)\right| & =\left|\sum\left(x_{i}, T \bar{\gamma}_{i}\right)\right|=\left|\sum\left(\bar{\gamma}_{i}, S x_{i}\right)\right| \\
& =\left|\sum\left(\gamma_{i} * S x_{i}\right)(e)\right| \leqslant\left\|\sum \gamma_{i} * S x_{i}\right\| \\
& =\left\|S\left(\sum \gamma_{i} * x_{i}\right) \mid \leqslant\right\| S\|\| \sum \gamma_{i} * x_{i} \| .
\end{aligned}
$$

(iii) $\Rightarrow$ (i). By the Hahn-Banach Theorem there exists an $h \in K^{*}$ such that

$$
\left(\sum \gamma_{i} * x_{i}, h\right)=\sum\left(x_{i}, \phi_{\bar{\gamma}_{i}}\right)
$$

for all $\gamma_{i}$ and $x_{i}$. In particular, for every $\gamma \in \hat{G}$ and $x \in K,(x, \bar{\gamma} * h)=(\gamma * x, h)$ $=\left(x, \phi_{\bar{\gamma}}\right)$. Hence $\bar{\gamma} * h=\phi_{\bar{\gamma}}$ for all $\gamma$, and $\tilde{h}=\phi$.

2.7 Corollary. For every $T \in$ Mult $K^{*}$ there exists an $h \in K^{*}$ such that $T f=f * h\left(f \in L_{\mathrm{l}}(G)\right)$.

Proof. For every $T$ there is an $h$ for which $\tilde{T}=\tilde{h}$. Then $T f=f * h$ if $f$ is any trigonometric polynomial; hence, if $f \in L_{\mathrm{I}}(G)$.

For absolutely continuous $K$ this result was proved in [3; 5.2].

For Hilbert spaces we obtain from 2.7 and 1.2:

2.8 CoRollary. If $K$ is a Hilbert space, then $\phi \in \Pi_{\gamma} K_{\gamma}$ can be extended to a multiplier of $K$ if and only if $\Sigma\left\|\phi_{\gamma}\right\|^{2}<\infty$.

2.9 TheOREM. Let $K$ be absolutely continuous. Let $\phi \in \Pi_{\gamma}\left(K^{*}\right)_{\gamma}$ be so that the net $\left(\sum_{\gamma \in \Delta} \phi_{\gamma}\right)_{\Delta \in F}$ is bounded. Then this net is $w^{*}$-convergent. If $h$ is its $w^{*}$-limit then $\phi=\tilde{h}$ and

$$
(x, h)=\sum_{\gamma \in \hat{G}}\left(\tilde{x}_{\gamma}, \tilde{h}_{\bar{\gamma}}\right) \quad(x \in K) .
$$

Note. Apparently, here we have analogs of the inversion formula and the Parseval relation from the theory of Fourier transformation.

Proof. $\phi$ can be extended to a multiplier $T$ of $K^{*}$, and $T f=\sum f * \phi_{\gamma}$ for all $f \in L_{\mathrm{I}}(G)$. By 2.7 there is an $h \in K^{*}$ such that $T f=f * h$ for all $f$. Now every $x \in K$ can be written as $f * y$ for certain $f \in L_{1}(G)$ and $y \in K$. Then

$$
\begin{aligned}
(x, h) & =\left(y, f^{*} * h\right)=\left(y, T f^{*}\right)=\left(y, \Sigma f^{*} * \phi_{\gamma}\right) \\
& =\sum\left(y, f^{*} * \phi_{\gamma}\right)=\sum\left(x, \phi_{\gamma}\right) .
\end{aligned}
$$


Hence,

$$
h=w_{\Delta \in F}^{*} \lim _{\gamma \in \Delta} \sum_{\gamma} \phi_{\gamma} .
$$

For $\gamma \in \hat{G}, \phi_{\gamma}=T \gamma=\gamma * h$; so $\phi=\tilde{h}$. Further, for $x \in K$,

$$
\begin{aligned}
(x, h) & =\sum\left(x, \phi_{\bar{\gamma}}\right)=\sum\left(x, \bar{\gamma} * \phi_{\bar{\gamma}}\right) \\
& =\sum\left(\gamma * x, \phi_{\bar{\gamma}}\right)=\sum\left(\tilde{x}_{\gamma}, \tilde{h}_{\bar{\gamma}}\right) .
\end{aligned}
$$

A linear module homomorphism is simply called a homomorphism.

In this section $G$ is a compact abelian group of homeomorphisms of a locally compact Hausdorff space $X$, such that the mapping $(s, x) \mapsto s x(s \in G ; x \in X)$ is jointly continuous. We denote by $C(G), C_{0}(X), C_{00}(X)$ the spaces of all continuous functions on $G$, all continuous fuctions on $X$ vanishing at infinity, and all continuous functions on $X$ with compact supports, respectively. The formula

$$
(f * k)(x)=\int f(s) k\left(s^{-1} x\right) d s \quad\left(k \in C_{0}(X) ; x \in X\right)
$$

turns $C_{0}(X)$ into an absolutely continuous Banach $L_{1}(G)$-module. (For details, see [6].) We identify $C_{0}(X)^{*}$ with the Banach space $M(X)$ of all bounded Radon measures on $X$, writing $(k, \mu)$ instead of $\int k d \mu\left(k \in C_{0}(X), \mu \in M(X)\right)$. The induced module composition on $M(X)$ is given by

$$
(f * \mu)(Y)=\int f(s) \mu\left(s^{-1} Y\right) d s
$$

where $f \in L_{1}(G), \mu \in M(X), Y \subset X, Y$ a Borel set.

3.1 TheOREM. Let $T: C(G) \rightarrow M(X)$ be a homomorphism. Assume that $T f \geqslant 0$ whenever $f \in C(G)$ and $f \geqslant 0$. (Such a homomorphism $T$ is called positive.) Then there exists a $\mu \in M(X), \mu \geqslant 0$ such that

$$
T f=f * \mu \quad(f \in C(G)) .
$$

Thus, $T$ can be extended to an element of Mult $M(X)$.

Proof. If $\nu \in M(X), \nu \geqslant 0$, then

$$
\|\nu\|=\nu(X)=\int \nu\left(s^{-1} X\right) d s=(1 * \nu)(X)=\|1 * \nu\| .
$$

Thus, if $f \in C(X), f \geqslant 0$, then $\|T f\|=\|1 * T f\|=\|T(f) * 1\|=\|f * T 1\| \leqslant$ $\|f\|_{1}\|T 1\|$. For an arbitrary $f \in C(G)$ we can write $f=f_{1}-f_{2}+i f_{3}-i f_{4}$ where 
$f_{j} \in C(G)$ and $0 \leqslant f_{j} \leqslant|f|$ for each $j$. It follows that $\|T f\| \leqslant 4\|f\|_{1}\|T 1\|$. Therefore $T$ has a unique continuous linear extension $T_{1}: L_{1}(G) \rightarrow M(X)$. By continuity, $T_{1} \in$ Mult $M(X)$. According to Corollary 2.7 there exists a $\mu \in M(X)$ such that $T_{1} f=f * \mu\left(f \in L^{1}(G)\right)$. To prove $\mu \geqslant 0$ take $j \in C_{0}(X), j \geqslant 0$ and let $\left\{u_{\imath}\right\}$ be an approximate identity of $L^{\prime}(G)$ such that $u_{\mathrm{t}} \in C(G)$ and $u_{\imath} \geqslant 0$ for each $\iota$. By the absolute continuity of $C_{0}(X), j$ can be written as $f * j^{\prime}$ where $f \in L_{1}(G), j^{\prime} \in$ $C_{0}(X)$. Then $(j, \mu)=\left(f * j^{\prime}, \mu\right)=\lim \left(u_{\imath} * f * j^{\prime}, \mu\right)=\lim \left(f * j^{\prime}, u_{\imath}^{*} * \mu\right)=$ $\lim \left(j, T\left(u_{\imath}^{*}\right)\right) \geqslant 0$. Thus $\mu \geqslant 0$.

For multipliers of $M(X)$ we can extend Bochner's Theorem [4; 33.3], [7; 1.4.3]. A function $\phi: \hat{G} \rightarrow M(X)$ is said to be positive definite if

$$
\sum_{i, j=1}^{n} c_{i} \bar{c}_{j} \phi\left(\gamma_{i} \gamma_{j}^{-1}\right) \geqslant 0
$$

for all positive integers $n$, all complex numbers $c_{1}, \ldots, c_{n}$ and $\gamma_{1}, \ldots, \gamma_{n} \in \hat{G}$.

3.2 TheOREM. Let $\phi \in \Pi_{\gamma} M(X)_{\gamma}$. Then $\phi$ is positive definite if and only if there exists $\mu \in M(X), \mu \geqslant 0$ such that $\phi=\tilde{\mu}$.

Proof. Let $\mu \in M(X), \mu \geqslant 0 ; c_{1}, \ldots, c_{n} \in \mathbf{C} ; \gamma_{1}, \ldots, \gamma_{n} \in \hat{G}$. Take $k \in C_{0}(X)$, $k \geqslant 0$. For every $x \in X$,

$$
\begin{aligned}
0 & \leqslant \int\left|\sum_{i} c_{i} \bar{\gamma}_{i}(s)\right|^{2} k\left(s^{-1} x\right) d x=\sum_{i, j} c_{i} \bar{c}_{j} \int \overline{\gamma_{i}(s)} \gamma_{j}(s) k\left(s^{-1} x\right) d s \\
& =\sum_{i, j} c_{i} \bar{c}_{j}\left(\bar{\gamma}_{i} \gamma_{j} * k\right)(x) .
\end{aligned}
$$

Hence

$$
\begin{aligned}
0 & \leqslant \sum_{i, j} c_{i} \bar{c}_{j}\left(\bar{\gamma}_{i} \gamma_{j} * k, \mu\right)=\sum_{i, j} c_{i} \bar{c}_{j}\left(k,\left(\bar{\gamma}_{i} \gamma_{j}\right)^{*} * \mu\right) \\
& =\left(k, \sum_{i, j} c_{i} \bar{c}_{j} \bar{\mu}\left(\gamma_{i} \gamma_{j}^{-1}\right)\right)
\end{aligned}
$$

and $\Sigma_{i, j} c_{i} \bar{c}_{j} \tilde{\mu}\left(\gamma_{i} \gamma_{j}^{-1}\right) \geqslant 0$.

Conversely assume $\phi$ to be positive definite. For every $k \in C_{0}(X), k \geqslant 0$, the scalar valued function $\gamma \mapsto\left(k, \phi\left(\gamma^{*}\right)\right)$ is positive definite. By Bochner's Theorem [4; 33.3] for such $k$ there exists a unique $\mu_{k} \in M(G)$ such that $\left(k, \phi\left(\gamma^{*}\right)\right)=\hat{\mu}_{k}(\gamma)$, $(\gamma \in \hat{G})$, and we have $\mu_{k} \geqslant 0$. The map $k \mapsto \mu_{k}$ can be extended to a linear positive, hence continuous, $U: C_{0}(X) \rightarrow M(G)$. Then

$$
\left(k, \phi\left(\gamma^{*}\right)\right)=(U k)^{\wedge}(\gamma) \quad\left(k \in C_{0}(X), \gamma \in \hat{G}\right) .
$$


It is easy to see that $(U(f * k))^{\wedge}=\hat{f}(U k)^{\wedge}=(f * U k)^{\wedge}$ for all $f \in L^{1}(G), k \in$ $C_{0}(X)$. Thus $U$ is a homomorphism. $U$ in turn induces a positive homomorphism $T: C(G) \rightarrow M(X)$ by

$$
(k, T f)=(f, U k) \quad\left(f \in C(G), k \in C_{0}(X)\right) .
$$

Applying Theorem (3.1) we obtain a $\mu \in M(X), \mu \geqslant 0$ such that $T f=f * \mu$ for all $f \in C(G)$. In particular, $(k, \gamma * \mu)=(k, T \gamma)=(\gamma, U k)=(U k)^{\wedge}\left(\gamma^{*}\right)=(k, \phi(\gamma))$ for all $k \in C_{0}(X)$ and $\gamma \in \hat{G}$. It follows that $\phi=\tilde{\mu}$.

We specialize further and assume the existence of a positive Radon measure $m$ on $X$ that is invariant under the action of $G$. Then every $L_{p}(m)(1 \leqslant p \leqslant \infty)$ can be made into a group algebra module by

$$
(f * k)(x)=\int f(s) k\left(s^{-1} x\right) d x \quad\left(f \in L_{1}(G), k \in L_{p}(m)\right)
$$

for locally almost all $x \in X$ (see [1]). For $p<\infty, L_{p}(m)$ is absolutely continuous. The natural linear maps $L_{1}(m) \rightarrow M(X), L_{p}(m) \rightarrow L_{q}(m)^{*}\left(p^{-1}+q^{-1}=1\right)$ are isometric homomorphisms. We identify $L_{p}(m)$ and $L_{q}(m)^{*}\left(p>1, p^{-1}+q^{-1}=\right.$ 1).

R. Ryan [8] characterizes those Fourier-Stieltjes transforms of measures on $G$ that actually are Fourier transforms of elements of $L_{1}(G) \cap L_{R}(G)$. His theorem can be extended in the following way.

3.3 Theorem. Let $1<p \leqslant \infty, p^{-1}+q^{-1}=1$. Let $E=\left\{k \in C_{00}(X) \mid \tilde{k}_{\gamma} \neq 0\right.$ for only finitely many $\gamma \in \hat{G}\}$. Let $\mu \in M(X)$ and assume that there exists a number $c$ such that

$$
\left|\sum_{\gamma \in \hat{G}}\left(\tilde{k}_{\gamma}, \tilde{\mu}_{\bar{\gamma}}\right)\right| \leqslant c\|k\|_{q}
$$

for all $k \in E$. Then there exists a $g \in L_{1}(m) \cap L_{p}(m)$ such that $\mu=g m$ (that is, $\mu(A)=\int_{A} g d m$ for all Borel sets $\left.A \subset X\right)$.

Proof. If $k \in C_{00}(X)$ and $\beta \in \hat{G}$, then

$$
(\beta * k, \mu)=\sum_{\gamma \in \hat{G}}(\beta * k, \bar{\gamma} * \mu)=\sum_{\gamma \in \hat{G}}\left(\beta * k, \tilde{\mu}_{\bar{\gamma}}\right) .
$$

The elements of $E$ are finite sums $\Sigma \beta_{i} * k_{i}$. Hence

$$
(k, \mu)=\sum_{\gamma \in \hat{\sigma}}\left(k, \tilde{\mu}_{\bar{\gamma}}\right) \quad(k \in E) .
$$


By the isomorphism between $L_{q}(m)^{*}$ and $L_{p}(m)$ there is a $g \in L_{p}(m)$ such that

$$
(k, \mu)=(k, g) \quad(k \in E) .
$$

If we can prove that $g \in L_{1}(m)$, then $\mu$ and $g m$ are bounded regular measures and $(k, \mu)=(k, g m)$ for all $k$ in a dense subspace of $C_{0}(X)$; then $(k, \mu)=$ $(k, g m)$ for all $k \in C_{0}(X)$ and $\mu=g m$.

Take $k \in C_{00}(X)$; let $S$ be the support of $k$. For $A \subset X$ let $\xi_{A}$ be the characteristic function of $A$. For every positive integer $n$ let $f_{n}$ be a trigonometric polynomial on $G$ such that $\left\|f_{n}\right\|_{1} \leqslant 1$ and $\left\|f_{n} * k-k\right\|_{1} \leqslant 2^{-n}$. Then $\lim f_{n} * k=$ $k$, a.e. and $f_{n} * k \in E$. Further, $\left\|f_{n} * k\right\|_{\infty} \leqslant\left\|f_{n}\right\|_{1}\|k\|_{\infty} \leqslant\|k\|_{\infty}$, and $f_{n} * k=0$ outside the compact set GS. Thus, $\lim \left(f_{n} * k\right) g=k g$ a.e., and $\left|\left(f_{n} * k\right) g\right| \leqslant\|k\|_{\infty}$ $|g| \xi_{G S}$. As $g \xi_{G S} \in L_{1}(m)$ it follows by the Lebesgue Dominated Convergence Theorem that

$$
\begin{aligned}
\left|\int k g d m\right| & =\lim \left|\int\left(f_{n} * k\right) g d m\right| \\
& =\lim \left|\left(f_{n} * k, \mu\right)\right| \leqslant \sup _{n}\left\|f_{n} * k\right\|_{\infty}\|\mu\| .
\end{aligned}
$$

Thus,

$$
\left|\int k g d m\right| \leqslant\|\mu\|\|k\|_{\infty} \quad\left(k \in C_{00}(X)\right) .
$$

Now let $C \subset X$ be compact. Let $U$ be an open set containing $C$ and of finite $m$-measure. Let $h$ be a measurable function, $|h(x)| \leqslant 1$ for all $x$, such that $h g=|g| \xi_{C}$ and $h=0$ off $C$. For each positive integer $n$ choose $k_{n} \in C_{00}(X)$, $\left\|k_{n}-h\right\|_{1} \leqslant 2^{-n},\left\|k_{n}\right\|_{\infty} \leqslant 1, k_{n}=0$ outside $U$. By another application of the Lebesgue theorem (note that $g \xi_{U} \in L_{1}(m)$ ) we get

$$
\begin{aligned}
\int_{C}|g| d m & =\int|g| \xi_{C} d m=\int h g d m \\
& =\lim _{n} \int k_{n} g d m \leqslant\|\mu\| \sup _{n}\left\|k_{n}\right\|=\|\mu\| .
\end{aligned}
$$

As this is true for all compact $C$, it follows that $g \in L_{1}(m)$.

\section{References}

[1] S. L. Gulick, T. S. Liu and A. C. M. van Rooij, 'Group algebra modules, II,' Canad. J. Math. 19 (1967), 151-173.

[2] S. L. Gulick, T. S. Liu and A. C. M. van Rooij, 'Group algebra modules, III,' Trans. Amer. Math. Soc. 152 (1970), 561-579.

[3] S. L. Gulick, T. S. Liu and A. C. M. van Rooij, 'Group algebra modules, IV,' Trans. Amer. Math. Soc. 152 (1970), 581-596. 
[4] E. Hewitt and K. A. Ross, Abstract harmonic analysis I-II (Springer Verlag, Berlin, 1963, 1970).

[5] R. Larsen, The multiplier problem (Lecture Notes in Mathematics, 105, Springer Verlag, Berlin, 1969).

[6] T.-S. Liu, A. C. M. van Rooij and J.-K. Wang, 'Group representations in Banach spaces: orbits and almost-periodicity,' Studies and essays presented to Yu-Why Chen on his 60 th Birthday April 1, 1970, pp. 243-254 (Mathematical Research Center, National Taiwan University, Taipei, Taiwan, China, 1970).

[7] W. Rudin, Fourier analysis on groups (Interscience, New York, 1962).

[8] R. Ryan, 'Fourier transforms of certain classes of integrable functions,' Trans. Amer. Math. Soc. 105 (1962), 102-111.

[9] J.-K. Wang, 'Multipliers of commutative Banach algebras,' Pacific J. Math. 11 (1961), 1131-1149.

University of Massachusetts

Amherst, Massachusetts 01003

U.S.A.
Catholic University

Nijmegen

The Netherlands 